\title{
Improved Digital Image Restoration Algorithm Based on Criminisi
}

DAI Lei ${ }^{1 *}$, JIANG Dai-Hong ${ }^{1}$, DING Bin ${ }^{1}$, James $\mathrm{K} \mathrm{Hahn}^{2}$

${ }^{1}$ Department of Information and Electrical Engineering, Xuzhou Institute of Technology Xuzhou 221018,China

${ }^{2}$ Department of Computer Science, The George Washington University, Washington

DC 20052, USA

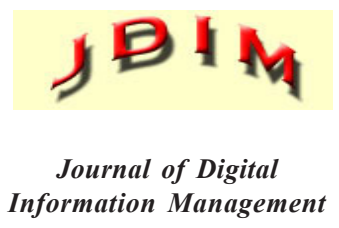

73829@163.com

ABSTRACT: Digital image inpainting pertains to the reconstruction of missing or damaged portions of images in a manner that is not detectable to the observer. This study evaluates the Criminisi algorithm for digital image inpainting and proposes four improvements. First, a new priority function was introduced using piecewise function to adjust the sequence of inpainting process and change the operation used from multiplication to addition under certain conditions. This adjustment improved the flexibility of the algorithm thereby avoiding incorrect filling sequence caused by rapid decay of data term. Second, Sobel operator instead of the traditional gradient direction was used to improve the computation of isophote. This approach first restored points on the isophote, which is beneficial to information fusion. Third, a new matching function was proposed and a new matching search method was employed to identify samples in the neighborhood of the damaged region based on similarity. This improvement created the nearest optimal matching block used for inpainting. Finally, a new formula of smoothing error propagation for updating confidence value was defined. This method ensured the correct sequence of synthesis from the periphery to the center. Experimental results show that this improved algorithm yields satisfactory inpainting results and improves repair efficiency.

Subject Categories and Descriptors

K.2.8 [Digital Image Processing]: Digital Image Inpainting; B.2.4 [Improved Arithmetic]: Algorithms

General Terms

Digital Image Inpainting, Algorithms
Keywords: priority, Sobel operator, nearest optimal matching block, confidence value, synthesis sequence.

Received: 12 December 2015, Revised 27 January 2016, Accepted 12 July 2016

DOI: $10.6025 / \mathrm{jdim} / 2016 / 14 / 5 / 302-310$

\section{Introduction}

Digital image processing developed rapidly with the increasing popularity of computers and other digital products. Digital image acquisition, storage, transmission, and use may result in defects in local information of image. For example, compression, transmission, and storage of digitally scanned images of damaged murals or old photographs may lead to information loss. Clients may also eliminate or hide specific information in the images. Digital image restoration technology was developed to address these problems.

Digital image inpainting, which is also known as image completion, produces visually plausible image interpolation of image regions. These regions have missing data caused by damage or occlusion. The development of digital image inpainting has been delayed, but it soon became a research hotspot in the field of image processing and computer vision. Inpainting primarily aims to achieve nondetectable adjustments of an image. Digital image inpainting has been used in various applications, such as in art restoration, computer animation, special effects, and virtual reality. Digital image inpainting is key to solving several problems in image processing, such as image compression, super resolution, and concealment of video 
error. Research on digital image inpainting can solve these problems and promote the development of this area.

\section{State of The Art}

Digital image inpainting algorithms are classified into two categories. The first method proposed by Bertalmio [1] is based on partial differential equation (PDE) and variational method. The algorithm is a simulation of the repair process of professional repair personnel. In this process, image restoration is completed by spreading the external information of the boundary of the area for repair to the internal region along normal direction of the contour line. Chan and Shen proposed a variational framework based on total variation (TV) to recover missing information [2]. A curvature-driven diffusion equation was also proposed to realize the connectivity principle, which does not hold in the TV model [3]. The outcome is a rough, unfocused boundary of the repaired area. Masno filled the damaged region with isophote information, but this process attained poor anti-noise ability [4]. Chan and Zhou improved reconstruction quality by changing the repair model and wavelet coefficient [5]. Other inpainting methods can improve the partial differential method to repair an image $[6,7,8,9]$. These algorithms fill the missing image region by diffusing information from the known region into the missing region at the pixel level. These methods yield good inpainting of the small missing region, but they fail to fill large missing or textured regions.

The second method is based on texture synthesis and is suitable for repairing large areas. The classic algorithm proposed by Criminisi [10] is based on the sample-based texture synthesis algorithm; this algorithm integrates the characteristics of the first image inpainting method to improve restoration effect. Several scholars used this algorithm to propose new ones. Tangd used image texture features for image segmentation to repair damaged images and inhibit error propagation [11]. NieE and Huang introduced a new calculation method to determine the repair order of the damaged region and achieve improved repair effect $[12,13]$. Perez examined the influence of sample size on repair results [14]. The adaptive template repair algorithm was proposed based on these improvements. A number of scholars proposed a series of adaptive algorithms using image information $[15,16,17,18,19]$. Fu Shaochun et al. used neighborhood characteristics to select sample size $[20,21,22]$. These algorithms achieved satisfactory repair texture, but they also introduce texture overlap. Some scholars proposed an image restoration algorithm based on texture transfer, but these algorithms have high computational cost [23]. Others proposed an image restoration algorithm based on matching blocks with natural repair effect [24], but they also have high computational cost. The present study aims to improves the Criminisi algorithm given its popularity and satisfactory repair effect. The Criminisi algorithm has four main defects. The first defect of this algorithm is priority calculation. The template data value of this algorithm will rapidly decline to zero as the filling process advances thereby resulting in unreliable calculation of priority. The sequence of the filling process may be inaccurate, which affects the final restoration effect. Second, repaired images with rich texture do not produce good effects because of the simple calculation of isophote. Third, this process uses a global search algorithm to identify the optimal matching block. This process will generate false matches and reduce filling speed. Lastly, the identical treatment of the original pixels of the damaged area and the filled pixel indicates the same reliability of the two categories of pixels. This process does not consider repair effect thereby resulting in compromised restoration effect. The algorithm should determine perfectly matched areas and fills. This process requires searching, matching, and pasting. Another issue is determining the priority of the block that will be restored. We proposed an improved algorithm based on Criminisi to address this issue.

The rest of this paper is organized as follows. Section 3 analyzes the Criminisi algorithm. We suggest four improvements in priority calculation, isophote calculation, search of matching area, and update of confidence value. We conduct experiment in Section 4 by applying the proposed method in the digital images. We then compare our results with those of other methods. Conclusions and directions for future work are given in Section 5.

\section{Methodology}

\subsection{Introduction of the Criminisi algorithm}

The Criminisi algorithm uses a sample-based texture synthesis method to copy the source pixel region of the matched image. The target region is then filled with the duplicated pixel. The target region contains the existing texture and structure information. The configuration information between regions can be obtained by changing the filling order of pixels. This algorithm solves the problem of simultaneously generated texture and structure information and maintains high-efficiency, which is an advantage of the original texture synthesis algorithm.

Figure 1(a) shows image I; the target region is denoted as $\mathrm{W}$, contour line is $\mathrm{dW}$, and source region is $\mathrm{f}(\mathrm{f}=\mathrm{I}-\mathrm{W})$. Figure 1(b) shows the default size of square template $\psi_{p}$ along the contour line within the $9 \times 9$ target region. Center point $P$ is located in contour line dW. Template $y_{p}$ should contain part of the synthesized pixels. Each pixel within the template has a color value and a confidence value. The confidence value indicates the filling state of that point, wherein 1 indicates that it has been filled and 0 means otherwise. Each template on the contour line has a temporary priority that determines the priority of each template to be filled. The filling process should gradually start along the contour line to ensure that the structural information can be firstly generated. After initialization, the following three steps are repeated until the fillings are completed. 


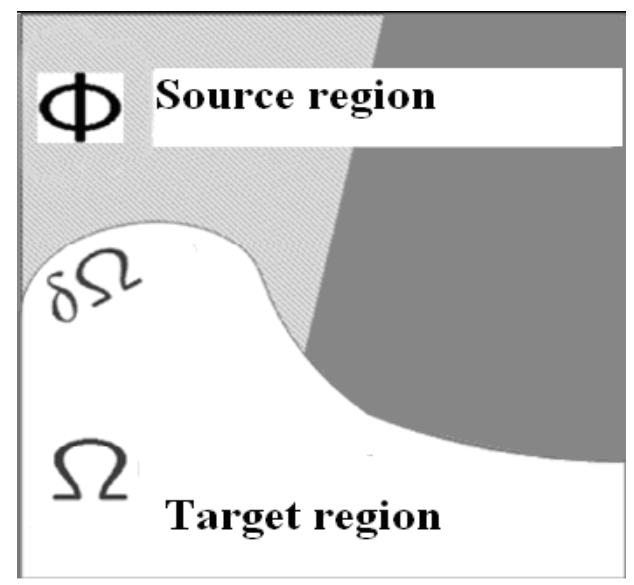

(a) Damaged image

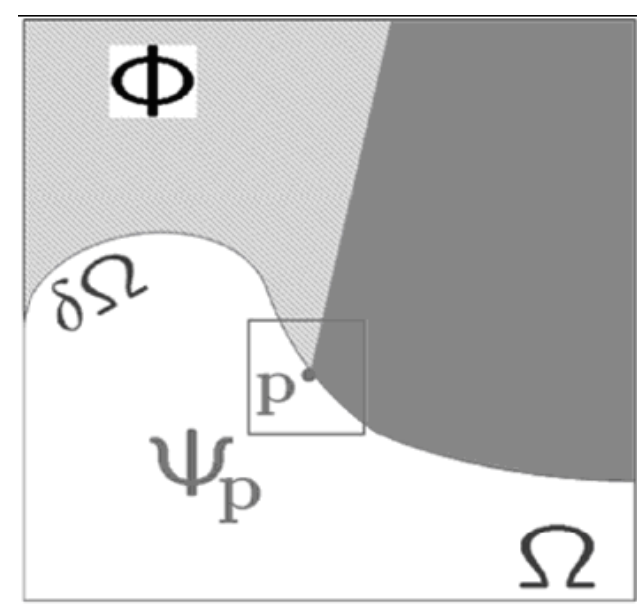

(c) Search block

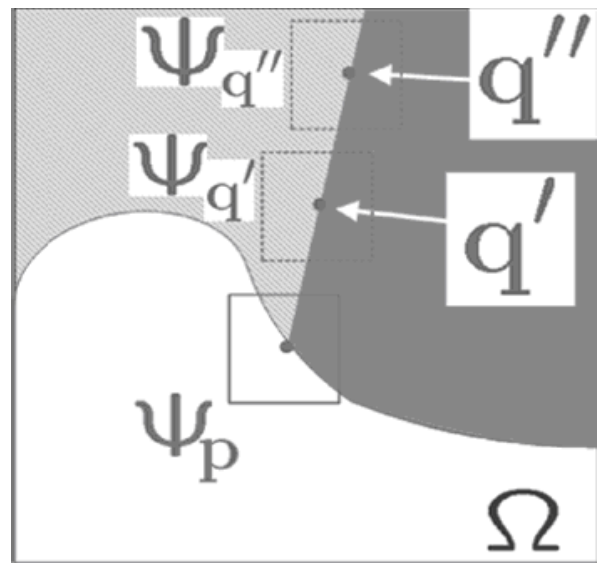

(b) Determine the block

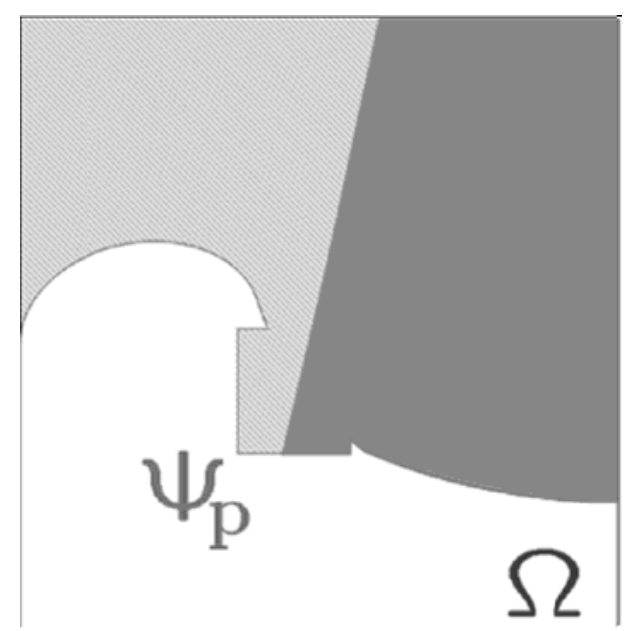

(d) Update

Figure. 1 Sample-based image inpainting

\subsubsection{Priority calculation}

Priority consists of two parts. The first part is the data value of the template, which reflects the information intensity of the image structure to ensure the preferred synthesis of the linear structure. The other aspect is the template confidence value, which shows that the template is filled with pixels with high credibility because the priority of this template rely on known pixels. The two aspects have mutual restraint.

Priority calculation adopts the following formula:

$$
P(p)=C(p) D(p)
$$

where

$$
C(p)=\frac{\sum_{q \varepsilon \psi_{p} \cap Q} C(q)}{\left|\psi_{p}\right|}, \quad D(p)=\frac{\left|\nabla_{I_{p}^{\perp} \cdot n_{p}}\right|}{\alpha}
$$

In Formula (2), $C(p)$ represents the confidence value of the template and $D(p)$ represents data value of the template. $C(q)$ represents the confidence value of the pixel at point q. During initialization, the value of each point in the target region should be 0 and the value of each point in in the source region is $1 .\left|\psi_{p}\right|$ represents the area of template $\psi_{p} ; \partial$ is a standardized parameter for the average grayscale, $\partial=255 . \boldsymbol{n}_{p}$ is the unit normal vector of the contour line at point $p$, and $\nabla I_{P}$ is the intensity and direction of isophote at point $\mathrm{p}$. Priority will be calculated first to obtain the synthetic sequence for each template. The formula shows that the confidence value of the template that contains a high number of filled pixels is large with high credibility. Templates at the tip of "peninsulas" of the filled pixels that jut into the target region are set aside until the majority of surrounding pixels are filled. The data values of templates with evident structure and projecting line segments are large. Thus, these templates are preferentially filled. Templates with large data values tend to approach prominent line segments, whereas templates with a high confidence values suppress the projection of line segments. Given these behaviors, a certain balance is achieved between the two.

3.1.2 Diffusion of texture and structure information 
After priority calculation, the template is filled according to priority. Samples are taken from the source region to identify the best match for the template. As shown in Figure 1 (c), the search covers the entire known information region to determine the template with minimal sum of color squared differences (SSD). This template is the optimal matching template. The corresponding pixel is then copied and filled into the template of the target area. As shown in Figure 1(d), complete filling includes texture and formed structure information. Unlike the traditional image inpainting algorithm, which disseminates information through diffusion, the proposed algorithm spreads information through sampling from the source region. This approach prevents blurring in the filling of the large area.

\subsubsection{Update of confidence value}

The confidence value of the template is updated after the remaining pixels within the template filling.

\subsection{Improvement of priority calculation}

The original algorithm determines priority based on the product operation. If the data item of the template is zero during restoration process, template restoration cannot be completed even if the confidence value is large because the priority is zero. Moreover, if $D(p)$ in more than one template is zero during restoration, their priority will also be zero thereby rendering $C(p)$ meaningless.

The use of $P(p)=C(p) D(p)$ to compute priority tends to be unreliable. Based on [24], the operation is changed to addition operation under the condition of $D(p) \neq 0$ and $C(p)$ $<0.5$. The following formula is then obtained:

$$
P(p)=\left\{\begin{array}{lc}
C(p) & D(p)=0 \\
\alpha C(P)+\beta D(p) & D(p) \neq 0 \text { and } C(p)<0.5 \\
C(p) D(p) & D(P) \neq 0 \text { and } C(p) \geq 0.5
\end{array}\right.
$$

In the formula, $\alpha$ and $\beta$ are adjustment parameters. $C(p)$ equal to zero is not taken into account because $p$ is located on the contour and the confidence value of the template that will be restored cannot be zero. This criterion guarantees that the priority will be high as long as confidence is sufficiently high when data item is zero. Gold segmentation points, such as $\alpha=0.382, \beta=0.618$, are used when the data item is not zero and confidence value is less than 0.5; this approach differs from that adopted in [24]. In the new algorithm, the data items are dominant factors and structure-preferred is restored. This approach is consistent with the principle of visual psychology. When the data item is not zero and the confidence value is greater than or equal to 0.5 , the Criminisi algorithm remains the first choice for calculating priority. This approach allows the two items to mutually inhibit each other, which ensures that restoration gradually spreads inward from the periphery. This method solves the problem of hysteresis of the restoration of low texture region using the Criminisi algorithm. This process addresses repair order that are inconsistent with the direction of linear structure.

\subsection{Improvement of isophote calculation}

In the Criminisi algorithm, the calculation of isophote $\nabla I_{p}^{\perp}$ is replaced with a simple gradient direction. This method does not effectively blend the isophote with the surrounding information in the face of images with rich texture and instead damages the final repair effect. The Sobel operator was employed to calculate the isophote and solve this issue. Templates measuring $3 \times 3$ were used with the Sobel operator.

In the following formula, $h$ represents the Sobel operator in the horizontal direction and $h$ ' represents the Sobel operator in the vertical direction.

$$
\nabla I_{p}^{\perp}=\left(g_{x}, g_{y}\right)^{\perp}=\left(\phi_{p}, h, \phi_{p}, h^{\prime}\right)^{\perp}
$$

In Formula (4), $\phi_{p}$ represents the $3 \times 3$ template with a center point on the boundary. Horizontal gradient $g_{x}$ is obtained by multiplying $\phi_{p}$ with horizontal Sobel operator $h$. Vertical gradient $g_{y}$ is obtained by multiplying $\phi_{p}$ with vertical Sobel operator $h$ '. The vertical orthogonal vector denotes the intensity of the isophote. The points on the isophote are restored first to retain the border line of the two color regions as the filling process continues. This approach maintain the linear structure of the image.

\subsection{Improvements in the matching region and optimal matching block}

Matching under the Criminisi algorithm is performed with known information from the entire image to determine the best matching block for the template with the highest priority on the contour line. The search space is composed of templates that are composed of pixels of the known region. This process is time consuming [22]. The information of the source image relevant to the template being restored only exists in a certain area. Based on the Markov Random Field model on texture locality and stability [20], the new algorithm narrows down the matching zone to the $S \times S$ square neighborhood with the pixel point that will be restored as the center pixel. The size of matching neighborhood $\mathrm{S} \times \mathrm{S}$ can be determined by the shape of the damaged area. Repeated testing and verification of a large number of images can maintain the size of the damaged area within $m \times n$. The new algorithm sets $\mathrm{K}=\min (\mathrm{m}, \mathrm{n})$ and $\mathrm{S}=2 \times \mathrm{K}+1$.

In the Criminisi algorithm, the order for searching the optimal matching block is from top to bottom and left to right. The algorithm searches for the candidate block in the matching area for blocks to be restored. SSD computation is implemented to identify the best matching block. The matching process creates more than one candidate block given the same minimum SSD of the block that will be restored. If the optimal matching block are far apart from blocks to be restored, it only ensure consistency with the features of known pixels in the block for restoration. 


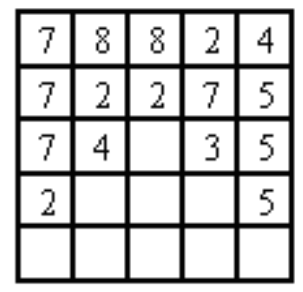

(a)To-be-restored block

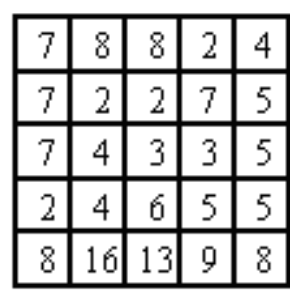

(b) Similar block 1

\begin{tabular}{|l|l|l|l|l|}
\hline 7 & 8 & 8 & 2 & 4 \\
\hline 7 & 2 & 2 & 7 & 5 \\
\hline 7 & 4 & 7 & 3 & 5 \\
\hline 2 & 2 & 7 & 4 & 5 \\
\hline 7 & 7 & 8 & 5 & 4 \\
\hline
\end{tabular}

(c) Similar block 2

Figure 2. Similar sample blocks

The characteristics of the remaining portion are likely inconsistent with expected results. The following matching block search will result in poor reproduction process once an error matching block is copied. Figure 2 shows as similar block with $5 \times 5$ measurement. Any block in Figure 2(b) and Figure 2(c) can be used to restore Figure 2(a). The repair effect of Figure 2(c)should be the best, so Figure 2 (c) is the real optimal matching block.

The matching in the Criminisi algorithm uses SSD calculation. Image texture is not considered. The color difference between the corresponding pixels of the block that will be restored and the selected matching block is only considered, not include the gradient difference of the corresponding position. The following matching function is proposed to solve the problem:

$$
\begin{aligned}
& \psi_{q}=\arg \min _{\psi_{q} \in \phi} d\left(\psi_{p}, \psi_{q}\right) \\
& d\left(\psi_{p}, \psi_{q}\right)=\sum_{i=1}^{m}\left(V_{i p}-V_{i q}\right)^{2}+\left(I_{i p}-I_{i q}\right)^{2}
\end{aligned}
$$

In Formula (5), $\psi_{q}$ represents the optimal matching block and $\psi_{p}$ represents the block to be restored. In Formula (6), $d\left(\psi_{p}, \psi_{q}\right)$ represents the distance between the to-berestored block and matching block, $\mathrm{m}$ represents the number of pixels in the block to be repaired, $V_{i p}$ represents the color value of the ith pixel in the to-be-restored block, $V_{i q}$ represents the color value of the ith pixel in the matching block, $I_{i p}$ represents the gradient value of the ith pixel in the to-be-restored block, $I_{i q}$, represents the gradient value of the ith pixel in the matching block. New matching function ensures a minimum difference in the color and texture of the two blocks.

A search method to identify the nearest, optimal matching block is proposed. The matching point of point $p$ for restoration is identified by using point $p$ as the center in the square neighborhood and by sequentially searching points whose chessboard distance to point $p$ are $n(1 \leq n$ $\leq \max , n \in N)$. These points are then used as matching points. This approach uses these points as center points and generates candidate blocks. Match computing is performed on these candidate blocks and block $\psi_{p}$ until the search is completed in the entire matching area. This method of searching the matching block is from near to distant, and choosing the best matching block which is the nearest one with a minimum distance difference in candidate blocks. The search records the candidate block with the minimum distance difference to the block that requires restoration. The block then copies this information to the appropriate location on the block for restoration. The repair result has a larger correlation with its neighborhood and is more consistent with the visual effects. According to some scholars [24], using a similar block more than once results in evidently human-induced restoration trace. To avoid this result, the logarithm of the average number of pixels in the optimal matching block is used as the cost function. Other studies [20] proposed the identification of the best matching points in the matching area before selecting the closest point for replacement. These methods share the common problem of excessive computational cost.

Results show that our method is more efficient than the above methods. Our method also achieved a natural image restoration effect.

\subsection{Improvement of confidence value update}

The confidence value of the restored pixel will be set to 1 when the Criminisi algorithm updates the template of a damaged image upon repair. Thus, in the next calculation of template $C(p)$, the $C(q)$ of the non-damaged pixels on the original image and the restored pixels are all 1 . This result demonstrates that the pixel after restoration and the non-damaged pixel on the original image are equally reliable. The algorithm updates the border after restoration of the template, which will likely cause the point with maximum priority on the new boundaries to lie within the just-filled template.

Incorrect information continues to extend to the interior. Unreasonable color information will cause error information to spread downward, which will eventually result in poor restoration effect. To overcome this weakness, the new algorithm sets a threshold value T. T depends on the value of distance difference corresponding to the specific template.

In Formula (7), $\mathrm{K}$ represents $d\left(\psi_{p}, \psi_{q}\right)$, as shown in Formula (6). The credibility of this template is high if $\mathrm{K}$ is 


$$
C(p)=\left\{\begin{array}{cc}
1 & K<T \\
\frac{\sum_{q \in \psi_{p} \cap \Omega} C(q)}{\left|\psi_{p}\right|} & K \geq T
\end{array}\right.
$$

smaller than threshold value T. The confidence value of the pixel that will be restored can be directly updated with that of the corresponding pixel of the source image template. Credibility is lower than that of the information in the source area if the value of $\mathrm{K}$ is greater than $\mathrm{T}$. Therefore, equal treatment is not suggested. The confidence value of the pixel to be restored is updated with the confidence value of template with the highest priority before matching. For example, if the confidence value of the template with the highest priority before matching is 0.68 , then the confidence values of the remaining pixels that will be restored after matching are
0.68. This process ensures that the deeper the pixel reaches the interior during constant patching, the lower the reliability will be. This finding is consistent with the general rule. Low reliability will result in low priority $P(p)$. Thus, the matching templates with substantially high confidence values should be employed. This process ensures that the synthesis sequence occurs from the periphery to the center.

\section{Analysis of Results And Discussion}

The experiments were conducted on a PC (Pentium Dual i5-4200M, 2.5 GHz, Memory 4 GB) using Visual C + + 6.0 .

In the algorithm, $\alpha=0.382, \beta=0.618$, and threshold $\mathrm{T}$ is 11. The restoration effects are compared in terms of the Criminisi algorithm, algorithm in [24], and our proposed algorithm.

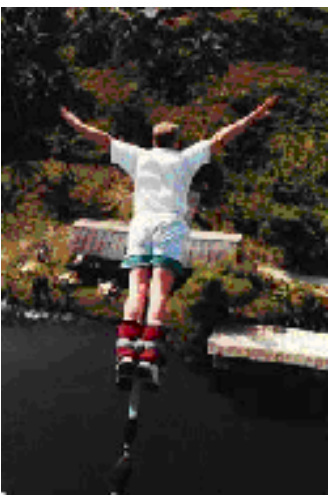

(a) Original image

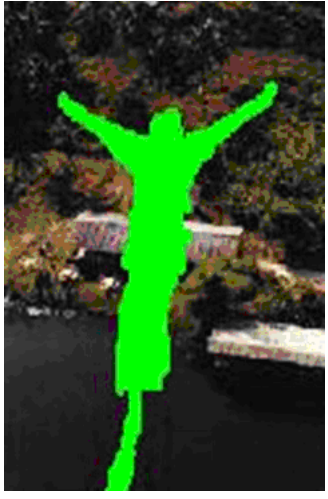

(b) Image for Restoratio

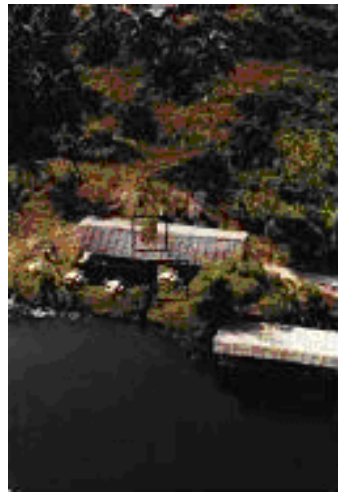

(c) Criminisi

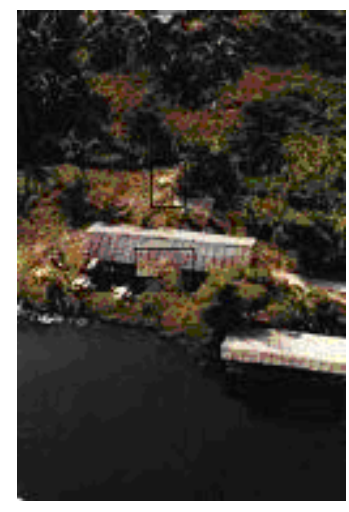

(d) Literature [24]

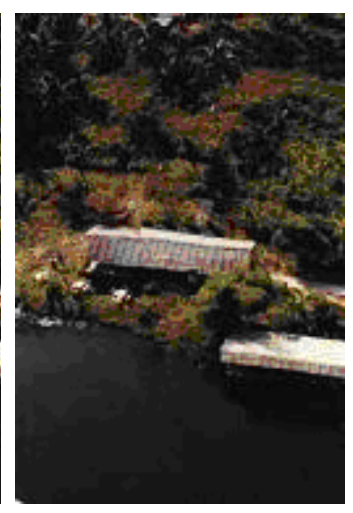

(e) Our proposed algorithm

Figure 3. Comparison of resulting images (“Bungee”)

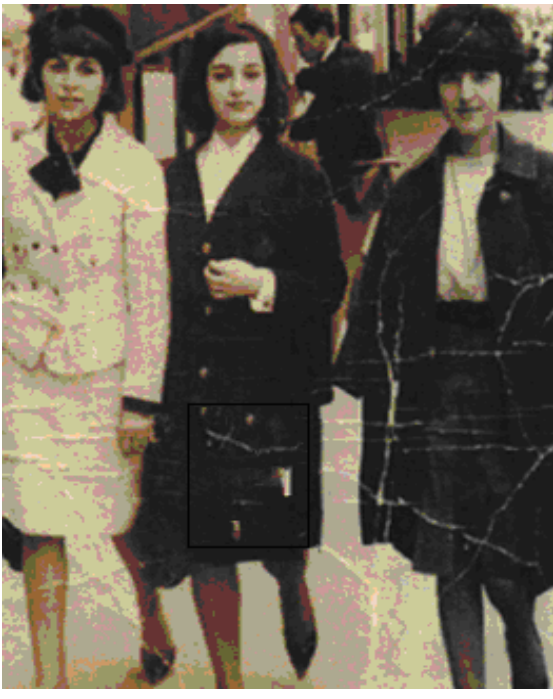

(a) Original image

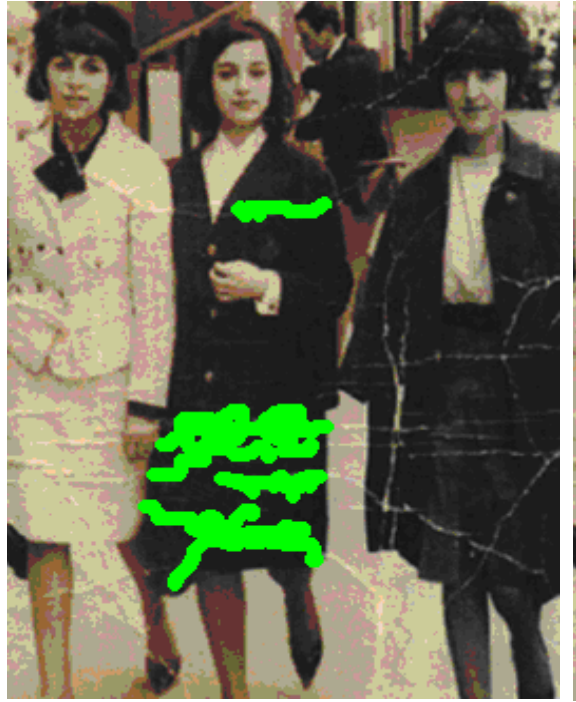

(b) Image for Restoration

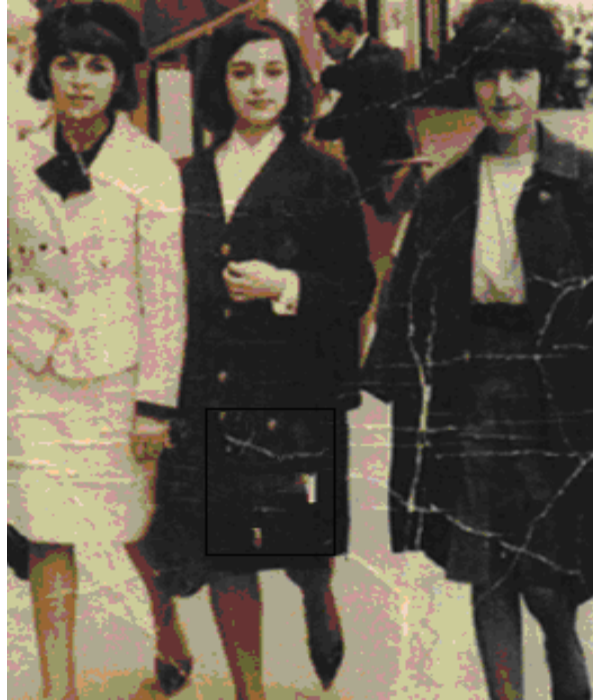

(c) Criminisi
Figure 3(c) indicates a clear fault in the roof area. Given the simple update of confidence value, the calculation of priority uses the product of data item and confidence item. Once an error message is filled, extremely high priority 


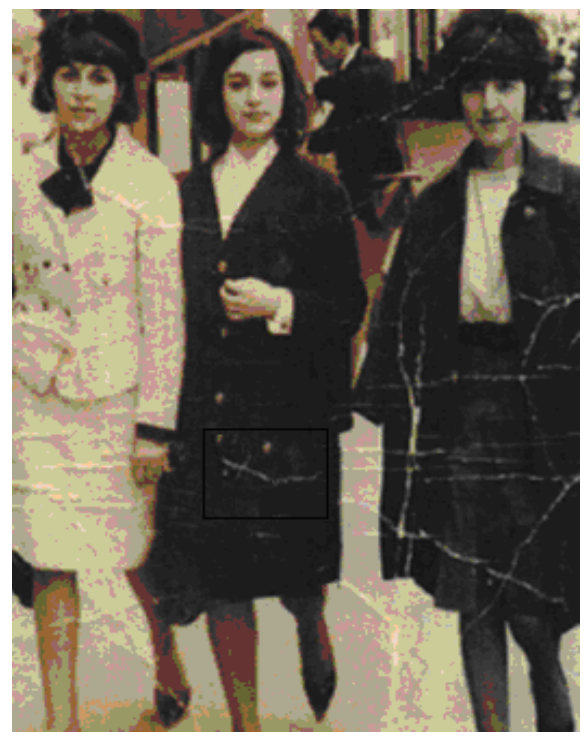

(d) Literature [24]

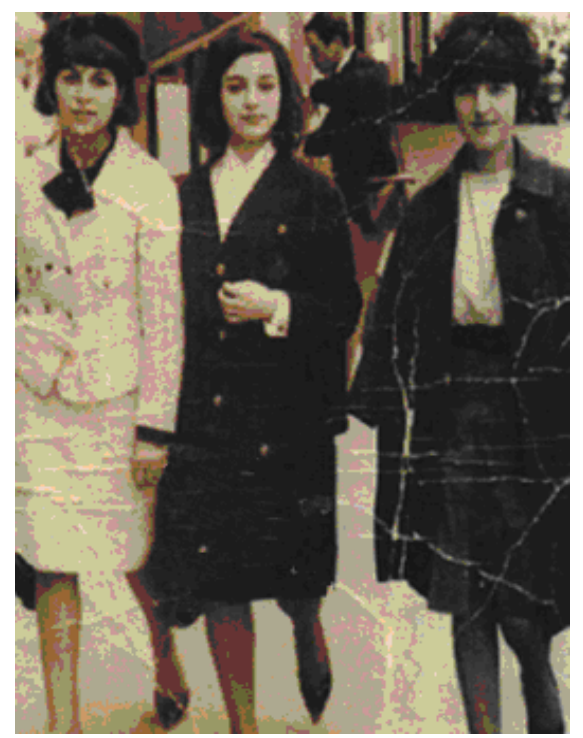

(e) Our proposed algorithm

Figure 4. Comparison of result images (“Girl”)

results in continuous extension of irrational color information. Needless "junk" exists in another marked area in the image because the Criminisi algorithm is a global search method. If the image contains noise, false matches often appear, and as the restoration progresses, repair errors increase.Figure $3(d)$ shows that the restoration effect of the algorithm in [24] demonstrated improvement. However, "junk" continues to exist in the identified area. Our algorithm is based on continuous improvement in priority calculation and optimal matching of block search. Figure 3(e) shows the effect of our proposed algorithm. This effect is natural and smooth and virtually zero flaws.

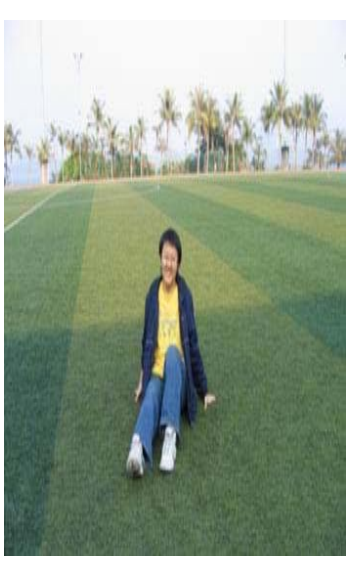

(a) Original image

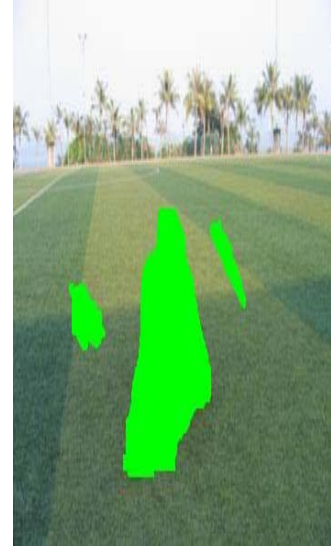

(b) Image for Restoration

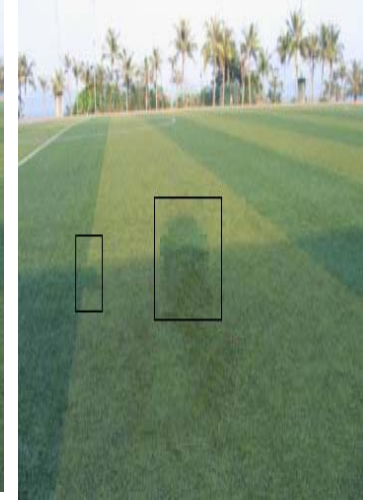

(c) Criminisi

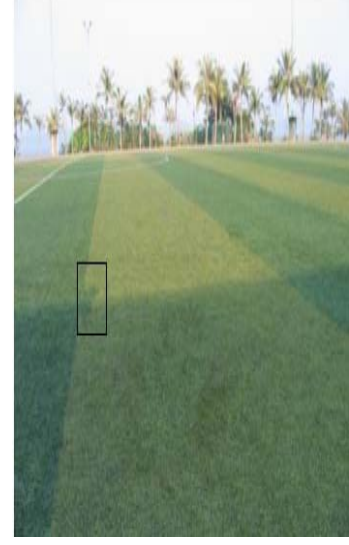

(d) Literature [24]

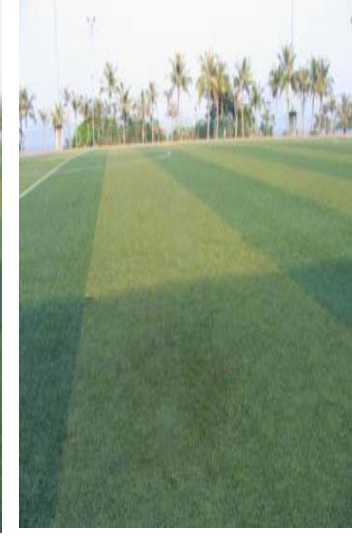

(e) Our proposed algorithm

Figure 5. Comparison of resulting images (“Grass”)

Three large damaged lawn patches are shown in Figure $5(b)$. These patches lie at the junction of a variety of color textures. This can better compare restore effects of the algorithms. Figure 5(c) indicates a clear restoration deviation in the identified region using the Criminisi algorithm. The algorithm in [24], which is shown in Figure 5(d), demonstrated significant improvement, except for the damaged area on the left with the junction of a variety of textures. The restoration effect of this algorithm is not natural. Our new algorithm uses the Sobel operator to improve the calculation of isophote value. Given this approach, the effect in Figure 5(e) is reasonable and consistent with real circumstances.

Peak signal-to-noise ratio (PSNR) is used as an objective evaluation standard of quality of image restoration. Its unit is $\mathrm{dB}$. Table 1 shows the PSNR of the image with three-color channels components and their specific data.

A high value of PSNR results in small error between the original image and the restored image. Table 1 shows that our algorithm is suitable for a variety of breakages with improved repair results. 
Table 2 shows the number of missing pixels in Figures 3 , 4 , and 5 and the duration of restoration of the three algorithms (in seconds). Experimental results shows that restricting the matching area to a square neighborhood centered on the pixel for restoration can significantly improve repair speed to ensure inpainting effect.

\begin{tabular}{|l|l|c|c|c|c|c|c|c|c|}
\hline \multirow{2}{*}{ Fig. } & \multicolumn{3}{|c|}{ Red channel } & \multicolumn{3}{c|}{ Green channel } & \multicolumn{3}{c|}{ Blue channel } \\
\cline { 2 - 10 } & Criminisi & Lit [24] & Proposed & Criminisi & Lit [24] & Proposed & Criminisi & Lit [24] & Proposed \\
\hline Fig.3 & 24.2167 & 25.9579 & 25.0937 & 24.1848 & 26.0728 & 25.1959 & 23.2630 & 25.2627 & 24.3996 \\
\hline Fig.4 & 37.8884 & 38.3325 & 39.0341 & 38.8608 & 38.9960 & 39.9049 & 40.5025 & 40.5924 & 41.6503 \\
\hline Fig.5 & 29.7048 & 29.9707 & 30.0186 & 30.6241 & 30.6037 & 30.6558 & 31.0850 & 31.3064 & 31.3677 \\
\hline
\end{tabular}

Table 1. PSNR of the restored image $\mathrm{db}$

\begin{tabular}{|c|c|c|c|c|c|}
\hline Fig & Image size & Number of missing Pixels & Criminisi & Lit[24] & Proposed \\
\hline Fig.3 & $206 \times 308$ & 7954 & 18 & 7 & 4 \\
\hline Fig.4 & $350 \times 262$ & 4255 & 19 & 5 & 3 \\
\hline Fig.5 & $350 \times 262$ & 8999 & 30 & 6 & 5 \\
\hline
\end{tabular}

Table 2. Comparison of computation time

\section{Conclusions}

Digital image inpainting has received considerable attention. The Criminisi algorithm is widely used as the mainstream algorithm of digital image inpainting. The present study applies the Criminisi algorithm to the field of image restoration. To overcome the deficiencies of this algorithm, we proposed four improvements, namely, priority calculation, isophote value calculation, search of matching region and the best matching block, and update of confidence value. The following conclusions are drawn:

(1) A new priority function is proposed based on the priority calculation malpractice of Criminisi algorithm. This new function is flexible and can be applied to a variety of situations using piecewise function. Product operation are changed to addition under certain conditions to avoid error in filling order.

(2) The Sobel operator is introduced in the calculation of isophote values to replace the gradient direction in Criminisi algorithm. The new method ensure that points on the isophote are restored first. The Sobel operator is conducive to the restoration of the linear structure of images with rich texture.

(3) A new matching search method of the nearest optimal matching block is proposed. This method narrows down the matching zone based on Markov random field model. A new matching function that considers additional matching texture information is employed. This approach can reduce error propagation caused by incorrect source patch matching. This algorithm is less time consuming because of its partial execution.
(4) The new confidence function results in varying confidence values in different conditions. Matching block selection in the known region can prevent expansion of error information. The experimental results show that the restoration effect of the improved algorithm is consistent with the characteristics of human visual system.

Restoration errors that are not consistent with human vision will continue to exist in complicated damaged images with intense structures. The universality of this algorithm has limited application in different types of image restoration. Thus, future work should further improve the universality and adaptability of this algorithm.

\section{Acknowledgements}

The study was supported by "Research on key techniques of image stitching and recognition in coal mining face, Jiangsu Province (333 Project) (No. BRA2014048)", Research on virtual image restoration algorithm based on sample, Xuzhou Science and Technology Project (No. KC15SH073)", and "Research on image virtual restoration algorithm in the protection of cultural relics, Xuzhou Institute of Technology Project (No. KC15SH073)".

\section{References}

[1] Bertalmio, M., Sapiro, G., Caselles, V., et al. (2000). Image Inpainting. In:The 27th Annual Conference on Computer Graphics and Interactive Techniques, p. 417424. New York, USA: ACM Press, July.2000.

[2] Chan, T., F., Shen, J., H.(2001). Nontexture Inpainting by Curvature-driven Diffusions. Journal of Visual Communication and Image Representation,12 (4) 436-449. 
[3] Chan, T., F., Kang, S., H., Shen, J., H.(2002 ). Euler's Elastica and Curvature-based Inpainting. SIAM Journal on Applied Mathematics, 63(2) 564-592.

[4] Masnou, S.(2002). Disocclusion: A Variational Approach Using Level Lines. IEEE Transactions on Image Processing, 11(2) 68-76.

[5] Chan, T., F., Zhou, H., M.(2007). Total Variation Wavelet Thresholding. Journal of Scientific Computing, 32(2) 315341.

[6] Xu, Z., Y., Lian, X., L., Feng, L., L.(2008). Image Inpainting Algorithm Based on Partial Differential Equation. In: 2008 ISECS International Colloquium on Computing, Communication, Control, and Management, pages 120124. Guangzhou, Chin: IEEE, August. 2008.

[7] Chen, Q., Montesinos, P., Sun, Q., S., et al. (2010). Ramp Preserving Perona-Malik model. Signal Processing, 90(6) 1963-1975.

[8] Wang, M., Q.,(2011). A Novel Image Inpainting Method Based on Image Decomposition. Procedia Engineering, 15(1) 3733-3738.

[9] Paul, G., Cardinale, J., Sbalzarini, I., F.(2013). Coupling Image Restoration and Segmentation: a Generalized Linear Model/Bregman Perspective. International Journal of Computer Vision, 104(1) 69-93.

[10] Criminisi, A., Perez, P., Toyama, K.(2004). Region Filling and Object Removal by Exemplar-based Image Inpainting. IEEE Trans Image Process, 13(9) 1200-1212.

[11] Tang, F., Ying, Y., Wang, J., et al.(2004). A Novel Texture Synthesis Based Algorithm for Object Removal in Photographs. In:The 9th Asian Computing Scienee Conference,pages 248-258.Chiang Mai, Thailand:Springer Berlin Heidelberg, December.2004.

[12] Nie, D., D., Ma, L., Z., Xiao, S., J.(2006). Similarity Based Image Inpainting Method. In:The 12th International Multi-Media Modelling Conference, p. 344-347.Beijing, China: IEEE, January.2006.

[13] Cheng, W., H., Hsieh, C., W., Lin, S., K., et al.(2005). Robust Algorithm for Exemplar-based Image Inpainting. In: The International Conference on Computer Graphic, Image and Vision, p. 64-69.Beijing,China: IEEE, July.2005.
[14] Perez, P., Gangnet, M., Blake, A.(2004). Patchworks: Example-based Region Tiling for Image Editing. Redmond,WA: Microsoft Research, MSR-TR-2004-04.

[15] Qin, C., Cao, F., Zhang, X., P.(2011). Efficient Image Inpainting Using Adaptive Edge-preserving Propagation. The Imaging Science Journal, 59 (4) 211-218.

[16] Sun, J., Yuan, L., Jia, J.(2005). Image Completion with Structure Propagation. ACM Transactions on GRAPHIC, 24 (3) 861-868.

[17] Yang, X., H., Wang, H., Q., Li, S., L.(2009). Image Inpainting Algorithm Based on the Adaptive Template and Update of the Confidence Term. Electronic Science and Technology, 22 (12) 69-72 (in Chinese).

[18] Wu, J., Y., Ruan, Q., Q.(2006). Object Removal By Cross Isophotes Examplar-based Inpainting. In:the 18th International Conference on Pattern Recognition, p. 810813.Washington, USA: IEEE, August .2006.

[19] Zhang, H., Y., Jin, Y., H., Wu, Y., D.(2010). Image Completion by a Fast and Adaptive Exemplar-based Image Inpainting. In:International Conference on Computer Application and System Modeling, p.115-119. Taiyuan, China: IEEE, October. 2010.

[20] Fu, S., C.,Lou, S., T.(2009). Regional Texture Synthesis Based Image Inpainting. Journal of Electronics \& Information Technology, 31(6) 1319-1322(in Chinese).

[21] Dai, S., M., Zhang, H., Y., Zeng, C.(2010). A Fast Algorithm of Exemplar Based Image Completion. Microcomputer \& Its Applications, 29 (22) 34-36(in Chinese).

[22] Choi, J., H., Hahm, C., H.(2013). An Exemplar-based Image Inpainting Method with Search Region Prior . In:2nd Global Conference on Consumer Electronics, p. 68-71. Tokyo, Japan: IEEE, October.2013.

[23] Lokhande, D., Zope, R., Bendre, V.(2014). Image Inpainting. International Journal of Computer Science and Network (IJCSN), 3 (1) 110-115.

[24] Wu, X., J., Li, G., Q.(2012). Large Scale Image Inpainting Based on Exemplar and Structure Information. Dianzi Xuebao(Acta Electronica Sinica), 40 (8) 15091514(in Chinese). 